\title{
Construction Program of Visualized Technical Supply Chain
}

\author{
Xiangjun $\mathrm{He}$
}

Safety and Environment Engineering College of Capital University of Economics and Business, China

Keywords: logistics visualization, order visualization, inventory visualization, visualized supply platform, chain architecture plan

\begin{abstract}
According to the need of modern competition and the operation and management of the supply chain, this paper analyzes the frame and system structure of a visualized supply chain, further reveals the technologies on supply chain acquisition, integration of heterogeneous data, data analysis and processing and real-time data display, and puts forward the building plan of Visualized Technical Supply Chain Platform including logistics visualization, order visualization and inventory visualization. This platform can effectively improve the visualization level of supply chain logistics, cash flow and information flow as well as the intelligent level of management.
\end{abstract}

\section{Introduction}

With the increasingly fierce market competition in modern society, and diversification of customer needs and enhancement of the uncertainty of market, the competition between enterprises has gradually transformed into competition between supply chains. The constant change of modern business environment and market conditions makes the information communication between the supply of raw materials, product research and development and design, production, distribution and selling in the supply chain more frequent, and makes the integration depth of across-organization 
flows stronger. Supply chains always have the features such as autonomy, distribution, heterogeneity and parallelism during their operation. All of these features challenge the transparency of supply chain management [1].

For the above reasons, this paper intends to apply visualization technology into supply chain management, and puts forward visualized technology platform theoretically. Based on ERP system, the platform can take 3D model as the basic framework, establish database in each model to store attribute data and spatial data of the objects, acquire real-time data, and realize real-time display on 3D image and carrying out comprehensive analysis of the data, to realize the visualization, control and traceability of relevant indexes of supply chain and relevant node enterprises such as logistics, inventory and orders, which will effectively improve the transparency of whole supply chain, greatly reduce the risk of supply chain, and improve the efficiency of supply chain operation.

\section{Composition of Visualized Technical Supply Chain Platform and Key Technology to Realize It}

\section{Composition of visualized system of supply chain}

(1) Order visualization Order visualization refers to the visualization of whole order process of supply chain, including forecast, orders and collaboration of order modification, shipping and receiving, invoice and payment collaboration. It is mainly related to various information about the supply chain information flow, logistics and cash flow including orders, inventory records, confirmation letters, invoices, and quality information, etc.

The system can manage the orders between supply chain enterprises, and create, query, and confirm orders; and predict, shipping, receiving goods, invoicing, and payments, to complete the whole process of e-commerce among enterprises; can also carry out real-time tracking and warning. 
(2) Inventory visualization The system can display inventory status in real-time manner, and analyze and calculate storage period and storage amount and so forth. Through the visualization of inventories of nodes of supply chain enterprises, it can provide the latest detail on the goods in transit, being checked, returned or ordered, and give warning for shortage or excess of inventory and so forth.

(3) Logistics visualization Logistics mainly contains the physical movement of material from supplier to purchaser, including such links as goods transport, warehousing, transportation and handling, distribution, processing and relevant logistics information. The visualization of logistics status of nodes on the supply chain includes shipping, receiving, order tracking, shipment status tracking (including multilevel through transport and global logistics), reminder based on the rules, other order information matching or supporting the goods shipping and receiving. Logistics visualization system is primarily used to track goods logistics status in a real- time manner, and automatically count and show the progress of logistics.

(4) Performance monitoring system of visualized supply chain Through visualized data analysis on logistics, orders and inventory, it can build display platform, so as to calculate and summarize such indexes showing supply chain performance level as order execution rate, on-time logistics rate and inventory carry rate, and integrate them according to operational quality.

\section{Key Technologies Realized by Visualized Supply Chain Platform}

(1)Real-time data acquisition and display technology. The real-time data acquisition required for the visualization of the logistics, cash flow, information flow of the supply chain is the most basic work, which is realized mainly through barcode and RFID [2].

Barcode is a symbol composed of lines and blanks according to certain encoding rules to represent letters and digits and other data. Data acquisition can be realized through one-dimensional codes and two-dimensional codes according to different business requirements. The daily commodity barcode on the outer package belongs to one dimensional code. It has little information 
storage capacity to store a code, and can call the data in computer network through the code in use. Two-dimensional code has been developing in recent years. It can store more information with limited space, including text, images, fingerprints, signatures, etc., and can be used without help of computer.

In addition, RFID is an automatic non-contact identification technology. It can automatically identify targets through radio frequency signal and acquire relevant data. Its identification work needs no manual intervention. It can work in various severe environments. RFID technology can identify high-speed moving objects and can identify multiple tags at the same time with fast and convenient operation.

The most critical work is the display form of real-time data. Visualized supply chain platform finally needs to use rich charts, flexible analysis and striking abnormal prompts to show data. Multidimensional analysis diagrams include biaxial chart, radar chart (spider chart), polar diagram, and pie chart, etc. Key performance indexes can be displayed by using dashboard. This method is visual and understandable, and can use different colors to represent different degrees according to preset thresholds; give warning for excess and shortage of indexes. Once a threshold is surpassed or reached, alarm can be automatically given. Breakdown drawing can realize free jump from different angles and at different levels. It can decompose indexes, and track them according to decomposed index tree.

(2)Standard of heterogeneous data integration and integration technology selection. As a visualized supply chain platform involves different enterprises on the middle and lower reaches, and different businesses of procurement, selling and storing, the software and database used are diversified. The processing of heterogeneous data is a big technical problem. To make the platform open, firstly the internationally recognized standard format is adopted to realize the data exchange between relevant departments or between companies and enterprises and 
processing. For instance, EDI used for this platform is not simple data exchange between users, but sending and receiving messages in international format. These messages are directly sent and exchanged between the computer systems of receiver and sender. Entire transmission process is automatic without need of human intervention, reducing errors and improving efficiency. EDI workflow mainly includes generating EDI flat file, generating standard format file through translation, communication, and receiving and processing of EDI files [3].

Secondly, the data integration of heterogeneous platform is also complex. The data mapping and transformation system provide a key interface between the business information system in the company and the electronically related trade partners. The implementation of such complex system usually needs a lot of programming work, and needs multiple components to process different hardware and software platform, which is prone to becoming bottleneck of e-commerce system. The method of selecting integration technology involves not only the work efficiency, but also the advanced nature, openness and connectivity in the future. The platform selects internationally advanced GXS gateway as integration technology with following features:

GXS Application Integrator is a powerful software product with the functions like conversion between any data formats [4], application integration, GUI mapping and management. Its modular design and translation of data driving have unmatched flexibility, scalability and high performance. It can combine technologically advanced mapping, translation and integration system. As a universal data transformation and integration system, it is designed especially for the e-commerce environment and enterprise application integration (EAI) environment, and can receive and generate structured data in any format, including EDI, XML and data format special for application. Multiple applications can be integrated even without need of modifying current data format. GXS Application Integrator greatly reduces the workload of data integration. The Application Integrator adopting GXS can realize its integration by using modular 
components. That is, it can use its own different interfaces with heterogeneous databases and application systems. Single integration of each enterprise in the supply chain with it can make data of all enterprises linked, thus greatly reduce the workload of integration, and reduce the probability of errors. When a new enterprise joins the supply chain, it only needs an integration with the gateway. This gateway can satisfy all integration need including enterprise applications, database and data warehouse, artificial workflow, external business partners, Webservice, etc.

(3)Visualization processing technology. 1)ETL. ETL refers to the processes including Extract, Transform and Load, which is a key link in building a data warehouse. Data warehouse is a subjectoriented, integrated, stable and time-dependent changing data collection, and is used to support the decision making process during operation and management. Data warehouse system may have a lot of noise data. The main causes include overuse of abbreviations and idioms, data input error, duplicate records, lost values, and changes in the spelling. Even for a database that has been well designed and planned, if there is a lot of noise data, the system does make no sense. This is because the system cannot provide any support for decision- making and analysis system with "garbage in, garbage out" at all. To remove noise data, data in database system must be cleaned.

2)OLAP. OLAP (on-line analytical processing) is a software technology and can enable analysts to quickly, consistently and interactively observe information from each aspect, so as to deeply understand data. The information is directly transformed from original data and reflects an enterprise's real situation in the manner easiest for user.

\section{Building Plan of Visualized Technical Supply Chain Platform}

\section{Platform positioning and basic requirements Platform}

flexibility. 1)Platform flexibility is very important for the companies owning multiple platforms such as UNIX, NT and Windows 2000. The company needs to know that the software 
they select will not limit their selection for favorite computing platform. Application Integrator can be used on one platform.

Create mapping and run these mappings on other platforms. For instance, we can develop mappings in NT or Windows 2000 and run them in the UNIX production environment, vice versa. Only Application Integrator can provide you with truly cross-platform operation, and allow you not to deal with each product or Windows-based interface.

2)Openness . Openness allows integration with current and future system.

3)Diversified interfaces. XML and EDI with or user's proprietary data format can be directly integrated to current application environment, which is a unified method to integrate any message, application structure or XML and EDI standard.

Composition of platform system The software mainly includes:

Tool software to support running of hardware devices, such as software required for automatic control of experimental equipment, operating system required for server operating, firewall software, database software, etc., and software used to set up real-time hot backup between two servers in a bid to ensure data security.

The main platform of management software to support the operation of whole supply chain business, including supplier management, logistics management, customer management, distribution management, production management, etc.

Individual software to support some special link of supply chain, such as RFID barcode management software for data acquisition, WMS software to support goods flow of logistics 
center, and GPS, WMS software to support transport of goods.

\section{Integration plan of visualized supply chain}

Requirement for application integration (1)Integration standard. To realize the integration of heterogeneous system on visualized supply chain platform and thus exchange of commercial transaction data between different types of systems, the system integration interface needs to follow one of the data interchange standards below (EDI/ XML /ANSI X 12).

(2)Selection of integration mode. Integration modes mainly include mutual integration and centralized integration. This platform will adopt the second integration mode with the following advantages:

1)Less workload of integration. All nodes of supply chain only need integration with gateway. The gateway comes with a variety of data interfaces and can easily realize data connectivity with various application systems. In the gateway, it establishes the mapping between different data formats, which can be realized by dragging and dropping, so the workload of data integration is greatly reduced.

2)Good openness. Application of integrated gateway not only can solve the data integration between current systems. When a new supply chain member will join in the future, it only needs one integration with the application integration gateway, rather than repeated integration with other enterprises in the supply chain.

3)High flexibility. The platform independent nature of application integration gateway allow the mapping built on one platform by enterprise can be run on other platform, so enterprise application does not have to be confined to a kind of system 
platform, greatly enhances its flexibility.

Integration plan of visualized supply chain and its realization form 1)In the visualized order system, all modules are developed based on SOA architecture to realize coarse-grained and loosely coupled module encapsulation, thus facilitate the system's integration with current order management system (OMS). The method also facilitate the system's integration with external warehouse management system (WMS), transportation management system (TMS), electronic map service and RFID, thus enable the visualized order system to constantly have the functions of low cost and online real-time updating.

2)Visualized inventory integration plan with combination of RFID and GIS In the visualized inventory system, warehouse management system can apply the positioning technology with combination of RFID and GIS to tackle the problem that GPS or Compass and other positioning cannot be used in enclosed large warehouse. Using RFID card readers with rational distribution and fixed positions can dynamically identify the positions of RFID tags in the warehouse with positioning accuracy of meter level. GIS software can be used to visually display the spatial distribution of goods in the warehouse. Combination with RFID information management technology can realize intelligent scheduling and management of warehouse space, quickly locate goods, and carry out lean management of out-put and in-put of warehouse, so as to improve the efficiency of warehouse management.

3)Visualized logistics integration plan based on Internet of Things. In the visualized logistics system, latest 4G network technology can be used to greatly improve the real-time nature of monitoring, and through the combination with video technology and sensor technology to transmit a large amount of information such as video, temperature and pressure in real-time and online manner, improve the real-time nature of vehicle monitoring and its richness of content, tackle the real-time 
monitoring on the index status of vehicle as well as the real-time monitoring on the environment inside and outside the vehicle during transportation monitoring, avoid driver's fatigue driving, and timely find hidden danger. In addition, the technology can allow the command monitoring center to timely learn of the situation at scene in case of such accidents as traffic collision or leak of dangerous good, and make an accurate response.

4)Visualized supply system integration based on gateway and its concrete implementation plan Gateway can be used to realize direct application integration with application systems, namely, realize the data integration of ERP system with other ERP system, visualization platform, third party logistics, financial monitoring platform and the like; beside, according to mutual agreement, after above systems provide the interface data structure of application integration, gateway supplier can complete corresponding data transmission to achieve application integration; or use other methods to achieve application integration. In addition, it can obtain data from one system and transmit information to other systems; at last, according to the requirement of the system design, after information is transmitted to receiver through gateway integration software, it can assist in processing of receiving interface, and feed back the execution result.

\section{Functions realized by visualized supply chain platform} (1)Supporting modules and functions of visualized supply chain platform Provide open data interface, support acquisition of data on upper, middle and lower reaches in different ways, for instance, enterprises provide data for the platform to maintain; enterprises input data through the visualized platform; through the connection between the visualized platform and the enterprises' information systems to realize real-time data interaction and update;

Acquisition of the business and financial information of the enterprises on upper, middle and lower reaches of supply chain can 
realize the cash flow visualization function based on order visualization, inventory visualization and logistics visualization;

Set up the business model and event handling mechanism of supply chain, monitor important nodes in the supply chain, monitor the execution of supply chain, logistics and order to find and process abnormal operations to establish platform for the safe management of cash flow of inventory in supply chain.

(2)Information management module and function of supply chain. Led by core enterprises, from raw material supply to production, wholesale, retail and other supply chain nodes, it can analyze and manage the basic data, financial statements, personnel structure, operating conditions and other information of small and medium-sized enterprises on nodes, and carry out synchronous management on the demand of whole supply chain, the production plan, supply plan and sales plan of single enterprise on upper, middle and lower reaches.

(3)Performance monitoring module and function of visualized supply chain. At first, according to execution data modeling of visualized supply chain, through the monitoring and analysis on supply chain inventory quantity (cash flow), the monitoring and analysis on orders and the monitoring on on-time logistics rate by the visualized supply chain, it can carry out risk evaluation on the operation conditions of the whole supply chain, and build the performance evaluation system for the enterprises on nodes of supply chain based on this, to realize the risk control of the supply chain.

Second, it has the function of management cockpit. Use supply chain operation database to establish supply chain performance analysis model. In the forms of cockpit, use dashboard to show the credit record, financial indicators, loans, pledge management and other data relating to inventory management of the whole supply chain and its enterprises on 
lower, middle and upper reaches visually by applying reports, charts, and other analysis and processing tools, to establish basic platform for business and function analysis, evaluate the execution ability and health level of supply chain logistics and orders, evaluate the executive ability of the enterprises as the supply chain members, and provide support for decision-making through comprehensive analysis.

\section{Conclusion}

Building Plan for Visualized Technical Supply Chain Platform is proposed in this paper, and its functions are sorted and further expanded. It sets up visualized supply chain framework structure, analyzes the specific content to realize visualized supply chain. The platform manages logistics, information flow and cash flow based on the ERP information systems on upper and lower reaches, and with the help of communication connection with individual information systems, uniformly displays, manages and share the running status of whole supply chine in the manner of various graphics and data forms, so as to improve the degree of visibility and intelligent level of operation management of the supply chain system, and provide support for decision making regarding supply chain optimization. However, the plan is merely a theoretical research, deeper research and improvement will be needed during the actual construction process of the system.

\section{References}

[1] Zhang Fang. Application of visualization technology in supply chain management. Shanghai: Software College of Tongji University, 2006.

[2] Chen Dawen. Visualized management of supply chain based on Internet of things. Logistics engineering and management, $2011(03)$.

[3] Wang Gongcui, Lin Guanna NiuYubing. Application and development of EDI in e-commerce. Computer knowledge and technology, 2010, 6 (14): 3832-3833.

[4] Sharp VSCM visualized supply chain management platform, 
International Conference on Mechanical Science and Engineering (ICMSE2015)

China Information World, August 17, 2009, page 059. 